\title{
Readout demonstrator for a Large-Scale Pixel-Detector conforming to the ATLAS Phase-II Upgrade
}

\author{
Alessandro Gabrielli \\ On behalf of the ATLAS TDAQ Collaboration ${ }^{1}$ \\ University of Bologna and INFN Bologna \\ E-mail: alessandro.gabrielli@bo.infn.it
}

\begin{abstract}
After a long design and development, a prototype of the pixel detector front-end readout chip is proposed for the HL-LHC ATLAS and CMS upgrades. It provides data streams using up to four lanes running at $1.28 \mathrm{Gbps}$ each. In parallel to that, an off-detector high-speed electronic readout system card, namely FELIX, has been developed and proposed to be shared among the ATLAS sub-detectors. This paper describes in detail the implementation of a full readout chain for the front-end readout chip, namely the RD53A, using the current ATLAS Phase-II readout FELIX card. For this work the readout chain has required dedicated electronics as a hardware interface between the front-end chip and the off-detector readout. Moreover, to maximize the efficiency of testing the chip and to realize the readout chain, it is extremely important that, even for the first prototypes, the DAQ chain is as similar as possible to the final one. The implemented chain has been left available at CERN for further tests and developments for the entire ATLAS TDAQ collaboration. This readout chain is proposed as a general readout test-stand for Large-Scale Application of pixel detectors. Full details of the prototype and testing performed to date are presented.
\end{abstract}

${ }^{1}$ Copyright 2020 CERN for the benefit of the ATLAS Collaboration. CC-BY-4.0 license. 


\section{Introduction}

To support the increasing luminosity foreseen at the High Luminosity Large Hadron Collider (HL-LHC) [1] upgrade at CERN, all the main experiments will have to undergo a major upgrade in the following years. In particular, ATLAS (A Toroidal LHC AppartuS) TDAQ (Trigger and Data AcQuisition) is designing a shared electronics system to be used by different detectors, independent of the front-end data format and detector modularity. In this way the increasing data volumes can be supported by concentrating on a unique type of readout card that will interface with the diverse ATLAS sub-detectors. This readout approach is known as Front-End LInk eXchange (FELIX). The first implementation of this is under commissioning for the years 2019/2020 for the liquid argon calorimeter (LAr), New Small Wheel (NSW), Level-1 Calorimeter (L1Calo), inner barrel even sectors (BIS) 7/8 and a demonstrator for the Tile readout Phase-II Upgrade - described below - at CERN. The upgrade is scheduled to take place in 2020, including the production of over 100 custom I/O cards, known as FLX-712 [2] (see Figure 1) ready for data taking in 2021. As the FLX-712 is also planned to be used as a development board for the Run 4 FELIX, it was desirable to prototype next generation Pixel readout chip, known as RD53A [3, 4] using this board as the interface. However, at the time of the test, the RD53A and FLX-712 were not directly compatible because they use different data protocols and physical layers (voltage level, optical fibers, etc). An intermediate interface card, known as PIxel Detector high Luminosity UPgrade card ( $\pi$ LUP) [5, 6] has therefore been implemented. This card was designed and realized in Bologna to make the RD53A and FLX-712 components fully compatible (see Figure 2). The $\pi$ LUP features high-performance Xilinx Kintex and Zynq devices and a variety of input-output physical ports that make the card vary flexible and configurable depending on the firmware implementation.

The FLX-712 communicates via optical fibers (see Figure 3) through either 4.6 Gbps GigaBit Transceiver (GBT) optical link or 9.8 Gbps FULL Mode [7] protocols [8]. In contrast, the RD53A module communicates via Display Port (DP) connectors through $160 \mathrm{Mbps}$ Elink (input) and four lanes at $1.28 \mathrm{Gbps}$ using the Aurora $64 / 66$ protocol (output) [9]. The $\pi$ LUP handles both the FLX-712-to-RD53A slow-control data-path (downlink) and the RD53A-to-FLX-712 data-taking path (uplink). Concurrently, the $\pi$ LUP receives and decodes Aurora $64 / 66$ data from the RD53A chip, coming from the other four data lanes of the DP connector. Those four lanes,

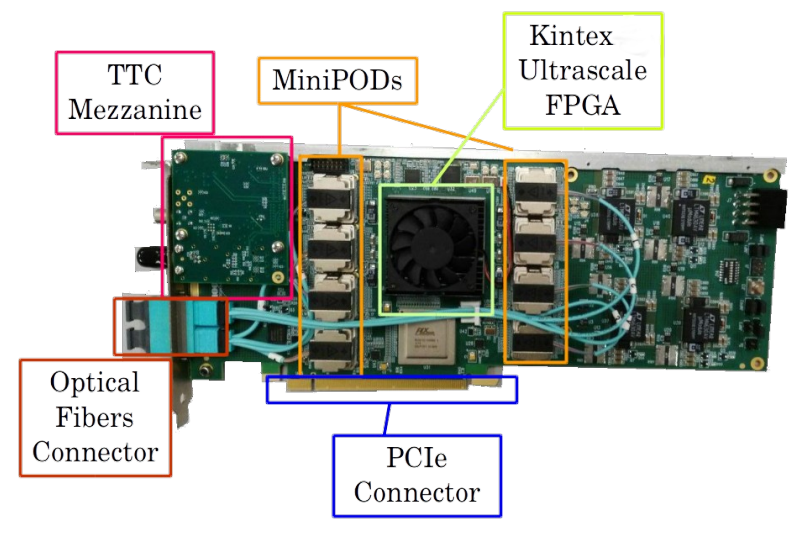

Figure 1: The FLX-712 Phase-I FELIX card. The Xilinx Kintex U1trascale FPGA, MiniPOD connectors, gen3 PCI Express connector, Timing and Trigger Control (TTC) mezzanine are shown.

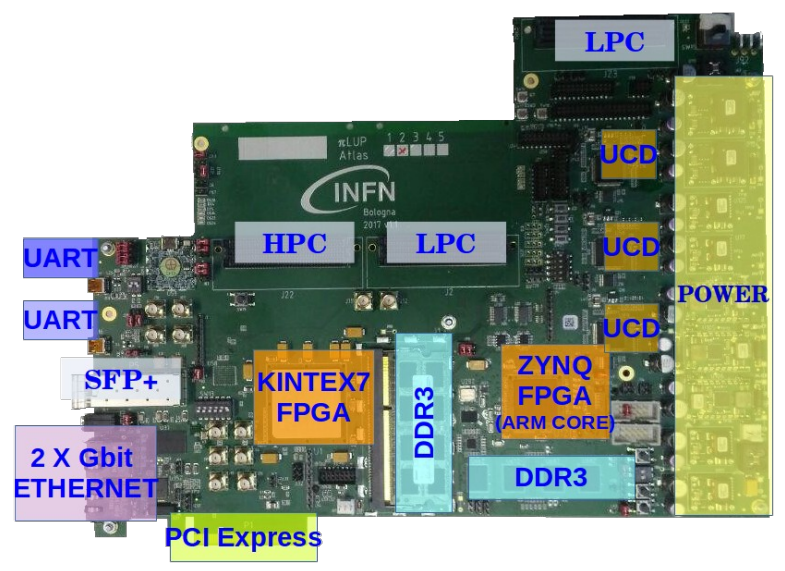

Figure 2: The $\pi$ LUP card featuring Xilinx Kintex FPGA, Xilinx Zynq FPGA, one High Pin Count (HPC) FPGA Mezzanine Card (FMC) connector, two Low Pin Count (LPC) FMC connectors, memory blocks (DDR3), a PCI Express connector, two Gbps Ethernet connectors, one Small Form-factor Pluggable (SFP) connector and two UART connectors.

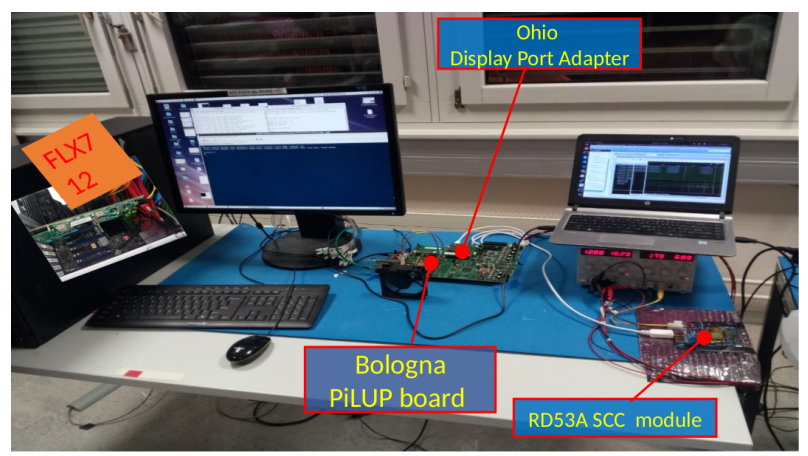

Figure 3: The RD53A readout chain showing the FLX-712 card (inside a PCIe server), $\pi$ LUP board acting as Protocol Converter and one front-end module card. To interface the $\pi$ LUP with the Display Port connector a FMC mezzanine has been used, called the Ohio Display Port Adapter. 
which each operate at $1.28 \mathrm{Gbps}$ data rate (resulting in a total throughput of $5.12 \mathrm{Gbps}$ ) are then passed to the so-called Protocol Converter firmware block (see Figure 4), which merges them into a single FULL Mode stream that is transmitted to FLX-712 via optical connection. This work has been crucial as a starting point for the design of a wider electronics upgrade that will be developed along with the future developments of the new front-end detectors. In Section 2 the main components of the ATLAS DAQ system and its upgrade are presented; Section 3 describes the RD53A readout chain prototype and some results and future developments.

\section{The ATLAS Upgrade}

Since the moment it was first started in 2008, the LHC at CERN has continued to constantly increase its center-of-mass energy and luminosity. In the next few years, the LHC will undergo two major series of upgrades meant to increase the centre-of-mass energy and the luminosity. The first upgrade, called the Phase-I upgrade, started at the end of 2018 and will be concluded in 2021, after which the LHC will have a period of datataking called Run 3 . The second major upgrade, called the Phase-II upgrade, will begin in 2025 and will conclude in 2027. After the second upgrade the LHC will enter a phase called high luminosity LHC. All the main experiments at CERN will be upgraded together with the accelerator, to improve their abilities to detect the particles generated after every collision and to sustain the harsher environmental conditions. Most of the ATLAS experiment sub-detectors will be upgraded during Phase-I or Phase-II. One of them, the ATLAS Inner Detector, will be completely redesigned during Phase-II, and a new version, called the Inner Tracker (ITk), will be installed during the upgrade. The ITk will be based on semiconductor technology and will be composed of pixel and silicon strip layers. The RD53A prototype of the on-detector readout front-end chip has already been produced and distributed to several institutes and laboratories to be tested before completion of the final design revision along with the real solid-state detector. As part of testing this version of the chip design it was therefore necessary to implement a complete readout chain.

\section{3. $R D 53 A$ readout chain}

The RD53A readout chain must involve the FLX-712 card and the software that will be used to read out the ITk during the ATLAS Phase-II upgrade. The RD53A front-end modules support an electrical interface based on Display port technology and data are encoded into Aurora 64/66b, while FELIX supports an optical fibrebased interface. The interconnection card between the front-end chip and the readout card is the $\pi$ LUP board, shown in Figure 2. It is an FPGA based card, implementing several connectors making it suited to address a broad variety of applications. In this context, the $\pi$ LUP board acts as a Protocol Converter, interfacing the front-end module chip and the FLX-712 card. The main firmware blocks are shown in Figure 4, in which two major data paths can be identified: configuration and data-taking. The first describes how configuration commands arrive from the readout software to RD53A. The commands are uploaded to the FLX712 card via the PCIe bus, and then are transmitted to the $\pi$ LUP board via GBT protocol (optical fiber). The $\pi$ LUP card decodes data from GBT protocol and reencodes them into a format that is compatible with the RD53A input, adding idle frames and sync frames when no configuration or trigger commands are sent. Finally the stream is propagated to RD53A via a $160 \mathrm{Mbps}$ serial line. During this stage, both the $\pi$ LUP card and the RD53A chip perform a clock-data-recovery operation, so that the system is fully synchronous with the Timing Trigger and Control (TTC) clock. The data-taking path describes how RD53A data are downloaded into the host PC. RD53A transmits data encoded into Aurora $64 / 66 \mathrm{~b}$ protocol to $\pi \mathrm{LUP}$ via four $1.28 \mathrm{Gbps}$ lanes. The $\pi$ LUP card performs data alignment and decodes the front-end data stream, that is then re-encoded into the 9.6 Gbps FULL Mode protocol. Data are then passed to FLX-712 via optical fibers and finally downloaded into the host server via the PCIe bus.

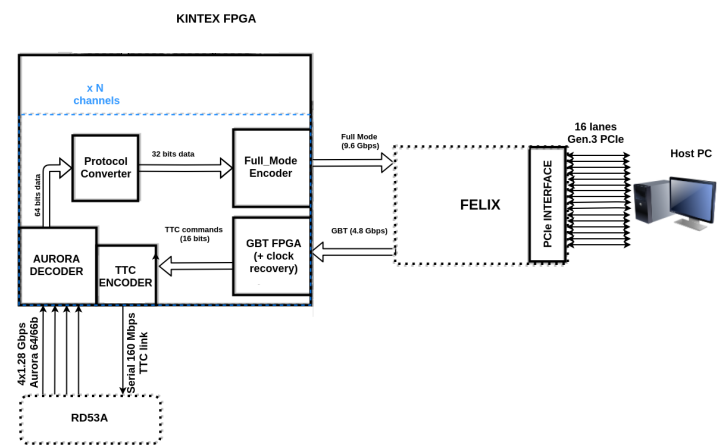

Figure 4: Firmware block design of the Protocol Converter.

Using this configuration, four front-end module chips - i.e. four RD53A chips - can be interfaced using a $\pi$ LUP and FLX-712 pair. To do so, the $\pi$ LUP card re- 
quires two FPGA Mezzanine Cards (FMC): a Display Port Adapter that features four DP connectors and a FM-S14 [10] mezzanine that implements four extra optical connectors.

Moreover, even though not discussed in this paper, the firmware of the $\pi$ LUP card also includes a kind of frontend emulator. In this way we are able to generate all the events necessary, according to the detector occupancy expectations, to fully test the readout channel before interfacing with the real detector front-end. In addition, by adapting the front-end data format, we can in principle emulate any kind of detector, not only the ITk.

As an example of the use of this test-stand some plots are shown below, taken from the chip calibrations that were investigated during the setup of the readout chain [11] . In particular, Figure 5 shows a Level-1 (L1) delay distribution obtained by keeping unchanged the injected charge and threshold for the entire matrix of $400 \times 192$ pixels, which was performed 100 times. In addition, Figure 6 shows a S-curve - the typical S-shaped curve originating from a circuit dependent on a threshold which represents a charge scan by keeping the threshold fixed. It is apparent that over a given charge the pixel starts registering a signal. This plot is created by reading one pixel out at a time across the entire matrix of the RD53A chip.

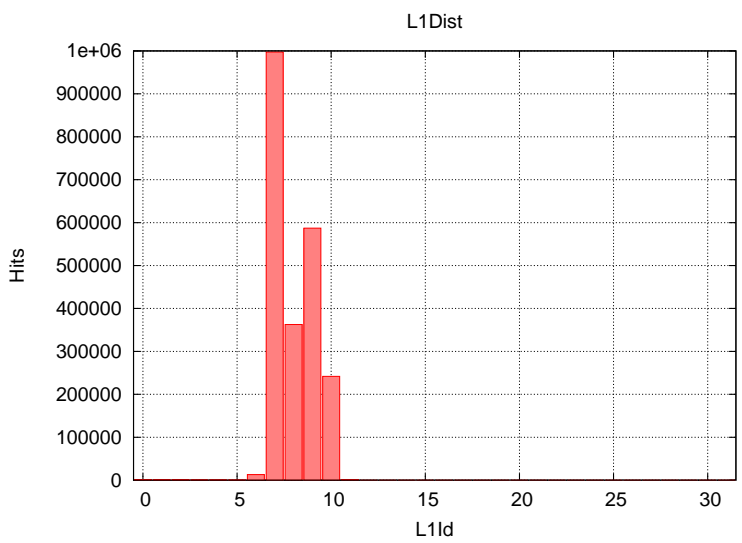

Figure 5: L1-delay count for the RD53A read out 100 times. X-axis refers to the ID numbers from 0 to 30 .

\section{Conclusions}

This work presents a prototype of the readout chain for the RD53A front-end chip using the FELIX system. The setup demonstrated the features of the chain, while at same time proving that the FELIX system is reliable and can be used to read out the

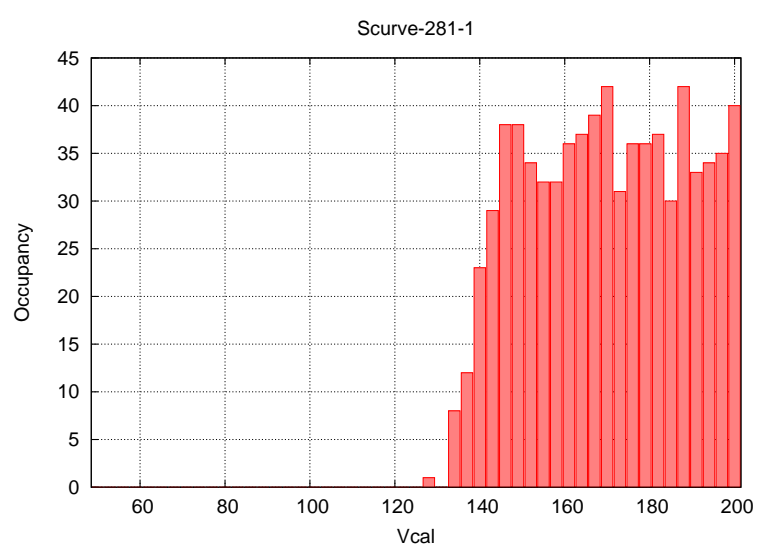

Figure 6: Charge-Threshold scan for individual RD53A pixels. Vcal is a register value used for calibrating the front-end voltage of the modules. The corresponding physical voltage depends on the module.

Phase-II Upgrade of the ATLAS sub-detectors. Also, the setup facilitated a first and fast development of all the software environment needed for the DAQ system. Using this software it is possible to perform front-end chip calibrations and digital and analog scans. Even if the main components of the described readout chain will not be used as-is in the ATLAS Phase-II DAQ system, the investigation process has made it possible to prepare for future implementations. Eventually, the RD53A chip will be replaced by newer and updated versions of the ASICs and the FLX-712 card will also be updated with new features. However, most of the design blocks realized and here presented as the firmware interface and Protocol Converter could be used or integrated into the final setup. Furthermore, since the entire readout chain is able to interface 4 front-end chips with a data rate of 5.12 Gbps each, which is $20 \mathrm{Gbps}$ in total, it can also be proposed as an example of a flexible high-speed readout system for a wide variety of future large-scale experiments.

\section{References}

[1] B. Schmidt, "The High-Luminosity upgrade of the LHC: Physics and Technology Challenges for the Accelerator and the Experiments", J. Phys. Conf. Ser., 2016, 706, 022002

[2] J. Anderson et al., "FELIX: A PCIe based high-throughput approach for interfacing front-end and trigger electronics in the ATLAS Upgrade framework", JINST 2016, 11, C12023

[3] E. Monteil, 'RD53A: a large scale prototype for HL-LHC silicon pixel detector phase 2 upgrades", 17-21 September 2018 Antwerp, Belgium, PoS(TWEPP2018)157

[4] M. Garcia-Sciveres, "The RD53A Integrated Circuit", CERNRD53-PUB-17-001

[5] N. Giangiacomi et al., "General purpose readout board

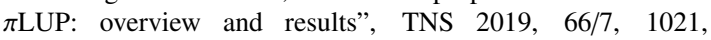
arXiv:1806.08858 [physics.ins-det]. 
[6] A. Gabrielli et al., "A multi-channel PCI Express readout board for fast readout of large pixel detectors", NIMA 2019, 924, 279

[7] W. Wu, "FELIX: the New Detector Interface for the ATLAS Experiment", arXiv:1806.10667v1 22 Jun 2018.

[8] P. Moreira et al., "The GBT, a Proposed Architecture for Multi$\mathrm{Gb} / \mathrm{s}$ Data Transmission in High Energy Physics", proc. of Topical workshop on electronics for particle physics (TWEPP 2007).

[9] Aurora 64B/66B v12.0, LogiCORE IP Product Guide, PG074 May 22, 2019

[10] Xilinx Part Number FM-S14

[11] K. Todome, "Data-acquisition system developments for ATLAS pixel QA/QC test toward High Luminosity LHC", 12th International Hiroshima Symposium (HSTD12), Hiroshima, Japan, 15 Dec. - 18 Dec. 2019, ID 333, ATL-ITK-SLIDE-2019-893. 